\title{
State Estimation for Continuous-Time Systems with Perspective Outputs from Discrete Noisy Time-Delayed Measurements
}

\author{
António Pedro Aguiar and João Pedro Hespanha
}

\begin{abstract}
This paper addresses the state estimation of continuous-time systems with perspective outputs, whose measurements arrive at discrete-time instants, are time-delayed, noisy, and may not be complete. Resorting to dynamic programming, we derive a minimum-energy estimator which produces an estimate of the state that is "most compatible" with the dynamics, in the sense that it requires the least amount of noise energy to explain the measured outputs. The state-estimator has the desired property that, under suitable observability assumptions, the estimate converges asymptotically to the true value of the state in the absence of noise and disturbance. In the presence of noise, the estimate remains bounded away from the true value of the state. We apply these results to the estimation of position and orientation for a mobile robot that uses a monocular chargedcoupled-device (CCD) camera mounted on-board to observe the apparent motion of stationary points. In the context of our application, the estimator can deal directly with the usual problems associated with vision systems such as noise, latency and intermittency of observations. Experimental results are presented and discussed.
\end{abstract}

\section{INTRODUCTION}

Consider a continuous-time system described by

$$
\begin{aligned}
\dot{x} & =A(u) x+b(u)+G(u) \mathbf{d}, \\
\alpha_{j} y_{j} & =C_{j}(u) x+d_{j}(u)+\mathbf{n}_{j},
\end{aligned}
$$

$j \in \mathcal{I}:=\{1,2, \ldots, N\}$, where $x \in \mathbb{R}^{n}$ denotes the state of the system, $u \in \mathbb{R}^{n_{u}}$ its input, $y_{j} \in \mathbb{R}^{m_{j}}$ its $j$ th perspective output, $\mathbf{d} \in \mathbb{R}^{n_{d}}$ an input disturbance that cannot be measured, and $\mathbf{n}_{j} \in \mathbb{R}^{m_{j}}$ measurement noise affecting the $j$ th output. Each $\alpha_{j} \in \mathbb{R}, j \in \mathcal{I}$ denotes a scalar that is determined by a normalization constraint such as

$$
\left\|y_{j}\right\|=1 \quad \text { or } \quad v_{j}^{\prime} y_{j}=1,
$$

where the $v_{j} \in \mathbb{R}^{m_{j}}$ denote constant vectors. We call (1)(2) a state-affine system with multiple perspective outputs, or for short simply a system with perspective outputs. These type of systems are inspired by the (single output) perspective systems introduced by Ghosh et al. [1].

Systems with perspective outputs typically arise when charged-coupled-device (CCD) cameras are used to acquire information about the position and orientation of moving rigid bodies. In Section $\mathrm{V}$ we address the pose estimation problem for mobile robots using measurements from a CCD camera mounted on-board that observes the apparent motion of stationary points. The dynamics of this system can be written as (1)-(2). The reader is referred to [1]-[3] for several other examples of perspective systems in the context of motion and shape estimation.

This material is based upon work supported by the National Science Foundation under Grants No. CCR-0311084 and ECS-0242798. The work of A. Pedro Aguiar was supported by a Pos-Doc Fellowship PRAXIS XXI from the Portuguese Foundation of Science and Technology.

A. Pedro Aguiar and João P. Hespanha are with Department of Electrical and Computer Engineering, University of California, Santa Barbara, CA 93106-9560, USA. \{aguiar, hespanha\}@ece.ucsb.edu
The problem of estimating the position and orientation of a camera mounted on a rigid body from the apparent motion of point features has a long tradition in the computer vision literature (cf., e.g., [4]-[9] and references therein). Filteringlike or iterative algorithms that continuously improve the estimates as more data (i.e., images) are acquired and that are robust with respect to measurement noise are especially desirable. Soatto et al. [6] formulates the visual motion estimation problem in terms of identification of nonlinear implicit systems with parameters on a topological manifold and propose a dynamic solution either in the local coordinates or in the embedding space of the parameter manifold. In [9], rigid-body pose estimation using inertial sensors and a monocular camera is considered. A local convergent observer where the states evolve on $S O(3)$ is proposed (the rotation estimation is decoupled from the position estimation). In the area of wheeled mobile robots, Ma et al. [10] address the problem of tracking an arbitrarily shaped continuous ground curve by formulating it as controlling the shape of the curve in the image plane. Observability of the curve dynamics is studied and an extended Kalman filter is proposed to dynamically estimate the image quantities needed for feedback control from the actual noisy images. An application for landing an unmanned air vehicle using vision in the control loop is described in [11]. In [7], the problem of navigation system design for autonomous aircraft landing based on measurements provided by airborne vision and inertial sensors is addressed. The authors cast the problem in a linear parametrically varying framework and solve it using tools that borrows from the theory of linear matrix inequalities. These results are extended in [12] to deal with the so-called out-of-frame events.

Returning to the system with perspective outputs (1)-(2), suppose that we acquire the measurements only at discrete times $t_{i}^{\prime}, i=0,1, \ldots, k$, with $t_{0}^{\prime}<t_{1}^{\prime}<\ldots<t_{k}^{\prime}$, and that we only have access to them after a time-delay $\tau_{i}$. Our sequence of measurements is therefore given by

$$
\mathbf{y}_{j}\left(t_{i}\right):=y_{j}\left(t_{i}^{\prime}\right)=y_{j}\left(t_{i}-\tau_{i}\right),
$$

where $\mathbf{y}$ denotes the time-delay observed variable, and $t_{i}=$ $t_{i}^{\prime}+\tau_{i}$. Furthermore, suppose that the measurements may not be complete, that is, at time $t_{i}^{\prime}$ only the outputs $y_{j}$ with $j \in \mathcal{I}_{i}$ were measured, where $\mathcal{I}_{i} \subseteq \mathcal{I}$ is an index set that is only the same as $\mathcal{I}$ if we acquire all the $N$ measurements at the time $t_{i}^{\prime}$.

The problem under consideration is to design an observer which estimates the continuous-time state vector $x(t)$ governed by equation (1), given the discrete time-delay measurements $\mathbf{y}\left(t_{i}\right)$ expressed by the output equation

$$
\begin{aligned}
\boldsymbol{\alpha}_{j_{i}} \mathbf{y}_{j}\left(t_{i}\right)= & C_{j}\left(u\left(t_{i}-\tau_{i}\right)\right) x\left(t_{i}-\tau_{i}\right)+d_{j}\left(u\left(t_{i}-\tau_{i}\right)\right) \\
& +\mathbf{n}_{j}\left(t_{i}-\tau_{i}\right), \quad j \in \mathcal{I}_{i},
\end{aligned}
$$


where $\boldsymbol{\alpha}_{j_{i}}$ is a normalization constraint such that (3) holds for $\mathbf{y}_{j}\left(t_{i}\right)$.

We propose a minimum-energy estimator that produces an estimate for the state of the perspective system that is "most compatible" with the system's dynamics and measured outputs. In particular, the optimal state estimate $\hat{x}$ at time $t$ is defined to be the value for the state that is compatible with the observations collected up to time $t$ and the dynamics of the system for the "smallest" possible measurement noise and disturbances, with "smallest" understood in an integral-square sense. Minimum-energy estimators were first proposed by Mortensen [13] and further refined by Hijab [14]. Game theoretical versions of these estimators were proposed by McEneaney [15]. It was recently shown by Krener [16] that this type of estimators is globally convergent when the system is observable for every input. In [17], it was shown that for projective systems with multiple inputs, convergence can be obtained under less restrictive observability assumptions. In [18], we improve upon the results in [17] by incorporating quadratic state-constraints in the minimum-energy formulation. State constraints allow one to take into account that some elements of the state must lie in given manifolds. In the context of rigid body motion, typically part of the state is a rotation matrix that is known to lie in $\mathrm{SO}(3)$, which can be expressed by quadratic constraints. In [18], the state estimates were used to close the loop and control a mobile robot to a desired position, defined with respect to visual landmarks. State-constraints for systems with perspective outputs also appeared in [3] in the context of motion estimation using a CCD camera and a laser range finder, where the measurements from camera and range finder were related by an algebraic constraint.

One of the main novelties of this paper is that we explicitly address the fact that the noisy measurements arrive at discrete-time instants, are time-delayed, and may not be complete. In this way, we can deal with usual problems associated to vision systems such as noise, latency, and intermittency. Resorting to dynamic programming, in Section III we derive a minimum-energy estimator. It has the desired property that, under suitable observability assumptions, the state-estimate converges asymptotically to the true value of the state in the absence of noise and disturbance. In the presence of noise, the estimate remains bounded away from the true value of the state (cf. Section IV). We can therefore use this state-estimator to design output-feedback controllers by using the estimated state to drive statefeedback controllers.

The theoretical results were experimentally validated by applying them to estimate the position and orientation of a mobile robot using measurements from an on-board CCD camera. The results obtained are discussed in Section V.

\section{Problem Statement}

Before we formulate the optimization problem, observe first from (1) that $x\left(t_{i}\right)$ satisfies

$$
\begin{aligned}
x\left(t_{i}\right)= & \Phi\left(t_{i}, t_{i}-\tau_{i}\right) x\left(t_{i}-\tau_{i}\right) \\
& +\int_{t_{i}-\tau_{i}}^{t_{i}} \Phi\left(t_{i}, \sigma\right)[b(u(\sigma))+G(u(\sigma)) \mathbf{d}(\sigma)] d \sigma
\end{aligned}
$$

where $\Phi\left(t, t_{0}\right)$ is the transition matrix of system (1) satisfying the differential equation $\dot{\Phi}=A(u) \Phi$. Therefore,

$$
\begin{aligned}
x\left(t_{i}-\tau_{i}\right)= & \Phi^{-1}\left(t_{i}, t_{i}-\tau_{i}\right) x\left(t_{i}\right)-\Phi^{-1}\left(t_{i}, t_{i}-\tau_{i}\right) \\
& \int_{t_{i}-\tau_{i}}^{t_{i}} \Phi\left(t_{i}, \sigma\right)[b(u(\sigma))+G(u(\sigma)) \mathbf{d}(\sigma)] d \sigma .
\end{aligned}
$$

Replacing this equation in (4) we get

$$
\boldsymbol{\alpha}_{j} \mathbf{y}_{j}\left(t_{i}\right)=\bar{C}_{j}(u) x\left(t_{i}\right)+\bar{d}_{j}(u)+\overline{\mathbf{n}}_{j}\left(t_{i}\right), \quad j \in \mathcal{I}_{i},
$$

where

$$
\begin{aligned}
\bar{C}_{j}(u) & :=C_{j}\left(u\left(t_{i}-\tau_{i}\right)\right) \Phi\left(t_{i}-\tau_{i}, t_{i}\right), \\
\bar{d}_{j}(u) & :=-\bar{C}_{j}(u) \int_{t_{i}-\tau_{i}}^{t_{i}} \Phi\left(t_{i}, \sigma\right) b(u(\sigma)) d \sigma+d_{j}\left(u\left(t_{i}-\tau_{i}\right)\right), \\
\overline{\mathbf{n}}_{j}\left(t_{i}\right) & :=-\bar{C}_{j}(u) \int_{t_{i}-\tau_{i}}^{t_{i}} \Phi\left(t_{i}, \sigma\right) G(u(\sigma)) \mathbf{d}(\sigma) d \sigma+\mathbf{n}_{j}\left(t_{i}-\tau_{i}\right) .
\end{aligned}
$$

The estimation problem can now be stated as follows:

Problem 1: Consider the continuous-time state equation (1) together with the discrete-time perspective output equation (5). Given an input $u$ defined on an interval $[0, t)$, and measured outputs $\mathbf{y}_{j}\left(t_{i}\right), j \in \mathcal{I}_{i}$ with $i=0,1, \ldots k$, $t_{0}:=0 \leq t_{1} \leq \cdots \leq t_{k} \leq t$, compute the estimate $\hat{x}(t)$ of the state at time $t$ defined as

$$
\hat{x}(t):=\arg \min _{z \in \mathbb{R}^{n}} J(z, t),
$$

where

$$
\begin{aligned}
& J(z ; t):=\min _{\substack{\mathbf{d}:[0, t), \overline{\mathbf{n}}_{j}\left(t_{i}\right), \boldsymbol{\alpha}_{j_{i}} \\
i=0,1, \ldots k}}\left\{\left(x(0)-\hat{x}_{0}\right)^{\prime} P_{0}\left(x(0)-\hat{x}_{0}\right)\right. \\
& +\int_{0}^{t}\|\mathbf{d}(\sigma)\|^{2} d \sigma+\sum_{i=0}^{k} \sum_{j \in \mathcal{I}_{i}}\left\|\overline{\mathbf{n}}_{j}\left(t_{i}\right)\right\|^{2}: \\
& x(t)=z, \dot{x}=A(u) x+b(u)+G(u) \mathbf{d} \\
& \left.\boldsymbol{\alpha}_{j_{i}} \mathbf{y}_{j}\left(t_{i}\right)=\bar{C}_{j}(u) x\left(t_{i}\right)+\bar{d}_{j}(u)+\overline{\mathbf{n}}_{j}\left(t_{i}\right)\right\}
\end{aligned}
$$

$P_{0}>0$ and $\hat{x}_{0}$ encodes a-priori information about the state.

The estimate $\hat{x}(t)$ can be interpreted as the value for which the measured outputs can be made compatible with the system dynamics (1) and (5) for the "smallest" possible noise $\overline{\mathbf{n}}_{j}$ and disturbance $\mathbf{d}$. This formulation considers the case when we do not have all the measurements at each time $t_{i}$ since $\mathcal{I}_{i}$ can be a strict subset of $\mathcal{I}$.

\section{THE OBSERVER EQUATIONS}

In this section we present the observer equations that are derived by using dynamic programming. In that framework, the function $J(z ; t), z \in \mathbb{R}^{n}, t \geq 0$ is viewed as a costto-go. In what follows, given a signal $x$ with a jump at time $t$, we denote by $x\left(t^{-}\right)$the limit of $x(\tau)$ as $\tau \uparrow t$ from below, i.e., $x\left(t^{-}\right):=\lim _{\tau \uparrow t} x(\tau)$. Without loss of generality we take all signals to be continuous from above at every point, i.e., $x(t)=\lim _{\tau \downarrow t} x(\tau)$. The following result solves Problem 1.

Theorem 1: The estimate $\hat{x}(t)$ of the state at time $t \geq 0$ defined by (6) and (7) can be computed as a solution to the impulse system defined by the initial conditions

$$
t_{0}=0, \quad P\left(t_{0}\right)=P_{0}, \quad \hat{x}\left(t_{0}\right)=\hat{x}_{0},
$$


the dynamic equations for $t_{i} \leq t<t_{i+1}, i=0,1, \ldots, k$

$$
\begin{array}{rlrl}
\dot{P}(t)= & -P(t) A(u)-A(u)^{\prime} P(t) & & \\
& -P(t) G(u) G(u)^{\prime} P(t), & & P\left(t_{i}\right)=P_{i} \\
\dot{\hat{x}}(t)= & A(u) \hat{x}(t)+b(u), & \hat{x}\left(t_{i}\right)=\hat{x}_{i}
\end{array}
$$

and the impulse equations at $t=t_{i+1}, i=0,1, \ldots, k-1$

$$
\begin{aligned}
P\left(t_{i+1}\right) & =P\left(t_{i+1}^{-}\right)+W\left(t_{i+1}\right), \\
\hat{x}\left(t_{i+1}\right) & =\hat{x}\left(t_{i+1}^{-}\right) \\
& -P\left(t_{i+1}\right)^{-1}\left[W\left(t_{i+1}\right) \hat{x}\left(t_{i+1}^{-}\right)+w\left(t_{i+1}\right)\right]
\end{aligned}
$$

where

$$
\begin{aligned}
W\left(t_{i+1}\right) & :=\sum_{j \in \mathcal{I}_{i+1}} \bar{C}_{j}^{\prime}(u)\left(I-\frac{\mathbf{y}_{j}\left(t_{i+1}\right) \mathbf{y}_{j}\left(t_{i+1}\right)^{\prime}}{\left\|\mathbf{y}_{j}\left(t_{i+1}\right)\right\|^{2}}\right) \bar{C}_{j}(u), \\
w\left(t_{i+1}\right) & :=\sum_{j \in \mathcal{I}_{i+1}} \bar{C}_{j}^{\prime}(u)\left(I-\frac{\mathbf{y}_{j}\left(t_{i+1}\right) \mathbf{y}_{j}\left(t_{i+1}\right)^{\prime}}{\left\|\mathbf{y}_{j}\left(t_{i+1}\right)\right\|^{2}}\right) \bar{d}_{j}(u) .
\end{aligned}
$$

Furthermore, the cost function $J(z ; t)$ defined in (7) is quadratic and can be written as

$$
J(z ; t)=(z-\hat{x}(t))^{\prime} P(t)(z-\hat{x}(t))+c(t),
$$

where $c(0)=0$ and, for all $i=0,1, \ldots k-1$,

$$
\begin{aligned}
c(t)= & c\left(t_{i}\right), \quad t_{i} \leq t<t_{i+1} \\
c(t)= & -\left(P\left(t^{-}\right) \hat{x}\left(t^{-}\right)+\hat{x}\left(t^{-}\right)^{\prime} P\left(t^{-}\right) x\left(t^{-}\right)+c\left(t^{-}\right)\right. \\
& -w(t))^{\prime}\left[P\left(t^{-}\right)+W(t)\right]^{-T}\left(P\left(t^{-}\right) \hat{x}\left(t^{-}\right)-w(t)\right) \\
& +\sum_{j \in \mathcal{I}_{i+1}} \bar{d}_{j}\left(I-\frac{\mathbf{y}_{j} \mathbf{y}_{j}^{\prime}}{\left\|\mathbf{y}_{j}\right\|^{2}}\right) \bar{d}_{j}, \quad t=t_{i+1}
\end{aligned}
$$

Proof: We start by proving (15). Take some $t \in$ $\left(t_{i}, t_{i+1}\right)$. Since $J(z ; t)$ is a cost-to-go it must satisfy the dynamic programming equation

$$
J_{t}(z ; t)=-\frac{1}{4}\left\|G^{\prime} J_{z}(z ; t)^{\prime}\right\|^{2}-J_{z}(z ; t)(A z+b),
$$

where $J_{t}$ and $J_{z}$ denote the partial derivatives of $J$ with respect to $t$ and $z$, respectively. For $k=0$, the value of $J(z ; t)$ can the be determined from the linear partial differential equation (18) with initial condition

$$
J(z ; 0)=\left(z-\hat{x}_{0}\right)^{\prime} P_{0}\left(z-\hat{x}_{0}\right), \quad z \in \mathbb{R}^{n}
$$

It turns out that the solution to (18)-(19) can be written as

$$
J(z ; t)=(z-\hat{x}(t))^{\prime} P(t)(z-\hat{x}(t))+c(t),
$$

for appropriately defined signals $\hat{x}(t)$ and $c(t)$. The signal $\hat{x}$ is then precisely the estimate for the state $x$ of the perspective system (1), (5). Moreover, matching (19) with (20) we conclude that $P(0)=P_{0}, \hat{x}(0)=\hat{x}_{0}, c(0)=0$. To verify that the solution to (18)-(19) can be written as (20), we replace this equation in (18), and obtain

$$
\begin{aligned}
z^{\prime}(\dot{P} & \left.+P A+A^{\prime} P+P G G^{\prime} P\right) z \\
& +2 z^{\prime}\left(-P \dot{\hat{x}}-\dot{P} \hat{x}-P G G^{\prime} P \hat{x}-A^{\prime} P \hat{x}+P b\right) \\
& +\dot{c}+2 \hat{x}^{\prime} P \hat{\hat{x}}+\hat{x}^{\prime} \dot{P} \hat{x}+\hat{x}^{\prime} P G G^{\prime} \hat{x}-2 \hat{x}^{\prime} P b=0 .
\end{aligned}
$$

This equation holds provided that

$$
\begin{array}{r}
\dot{P}+P A+A^{\prime} P+P G G^{\prime} P=0, \\
-P \dot{\hat{x}}-\dot{P} \hat{x}-P G G^{\prime} P \hat{x}-A^{\prime} P \hat{x}+P b=0, \\
\dot{c}+2 \hat{x}^{\prime} P \dot{\hat{x}}+\hat{x}^{\prime} \dot{P} \hat{x}+\hat{x}^{\prime} P G G^{\prime} \hat{x}-2 \hat{x}^{\prime} P b=0 .
\end{array}
$$

Replacing (21) in (22) and these two equations in (23), we conclude that (9)-(10) and (15) hold for $0 \leq t<t_{1}$. Observe also that $P(t)$ remains positive definite for all $0 \leq$ $t<t_{1}$. This can be verified by noting that the solution to (9) can be written as

$$
P=\Psi(0, t)^{\prime} P_{0} \Psi(0, t)+\int_{0}^{t} \Psi(\tau, t)^{\prime} P G G^{\prime} P \Psi(\tau, t) d \tau
$$

$t \geq 0$, where $\Psi(t, \tau)$ denotes the state transition matrix of $\dot{z}=\left(A+G G^{\prime} P\right) z$. Since $\Psi(t, 0) P_{0} \Psi(t, 0)^{\prime}>0$ and $P G G^{\prime} P \geq 0$, it follows that $P(t)>0$ for all $0 \leq t<t_{1}$.

Consider now the case $t=t_{k}, k>0$. From (7), we notice that $J\left(z ; t_{k}\right)$ can be written as

$$
\begin{aligned}
& J\left(z ; t_{k}\right)=\min _{\boldsymbol{\alpha}_{j_{k}}}\left\{\operatorname { m i n } _ { \substack { \mathbf { d } : [ 0 , t _ { k } ) , \boldsymbol { \alpha } _ { j _ { i } } \\
i = 0 , 1 , \ldots k - 1 } } \left\{\left(x(0)-\hat{x}_{0}\right)^{\prime} P_{0}\left(x(0)-\hat{x}_{0}\right)\right.\right. \\
& \quad+\int_{0}^{t_{k}}\|\mathbf{d}(\sigma)\|^{2} d \sigma+\sum_{j \in \mathcal{I}_{k}}\left\|\boldsymbol{\alpha}_{j_{k}} \mathbf{y}_{j}\left(t_{k}\right)-\bar{C}_{j} x\left(t_{k}\right)-\bar{d}_{j}\right\|^{2} \\
& \quad+\sum_{i=0}^{k-1} \sum_{j \in \mathcal{I}_{i}}\left\|\boldsymbol{\alpha}_{j_{i}} \mathbf{y}_{j}\left(t_{i}\right)-\bar{C}_{j} x\left(t_{i}\right)-\bar{d}_{j}\right\|^{2}: \\
& \left.\left.\quad x\left(t_{k}^{-}\right)=x\left(t_{k}\right)=z, \dot{x}=A x+b+G \mathbf{d}\right\}\right\} \\
& =\min _{\boldsymbol{\alpha}_{j_{k}}}\left\{J\left(z ; t_{k}^{-}\right)+\sum_{j \in \mathcal{I}_{k}}\left\|\boldsymbol{\alpha}_{j_{k}} \mathbf{y}_{j}\left(t_{k}\right)-\bar{C}_{j} x\left(t_{k}\right)-\bar{d}_{j}\right\|^{2}\right\}
\end{aligned}
$$

For $k=1$ we already saw that $J\left(z, t_{1}^{-}\right)$is given by (20). Assuming that it has the same form at time $t_{1}$, replacing it in the left and right-hand-side of (25), after some algebraic manipulation, we obtain

$$
\begin{aligned}
z^{\prime}\left[P\left(t_{k}\right)\right. & \left.-P\left(t_{k}^{-}\right)-W\left(t_{k}\right)\right] z \\
& +2 z^{\prime}\left[-P\left(t_{k}\right) \hat{x}\left(t_{k}\right)+P\left(t_{k}^{-}\right) \hat{x}\left(t_{k}^{-}\right)-w\left(t_{k}\right)\right] \\
& +c\left(t_{k}\right)+\hat{x}\left(t_{k}\right)^{\prime} P\left(t_{k}\right) x\left(t_{k}\right)-\hat{x}\left(t_{k}^{-}\right)^{\prime} P\left(t_{k}^{-}\right) x\left(t_{k}^{-}\right) \\
& -c\left(t_{k}^{-}\right)-\sum_{j \in \mathcal{I}_{k}} \bar{d}_{j}\left(I-\frac{\mathbf{y}_{j} \mathbf{y}_{j}^{\prime}}{\left\|\mathbf{y}_{j}\right\|^{2}}\right) \bar{d}_{j}=0
\end{aligned}
$$

where the definitions (13) and (14) were used. This equation for $k=1$ holds provided that

$$
\begin{aligned}
& P\left(t_{k}\right)-P\left(t_{k}^{-}\right)-W\left(t_{k}\right)=0, \\
& -P\left(t_{k}\right) \hat{x}\left(t_{k}\right)+P\left(t_{k}^{-}\right) \hat{x}\left(t_{k}^{-}\right)-w\left(t_{k}\right)=0, \\
& c\left(t_{k}\right)+\hat{x}\left(t_{k}\right)^{\prime} P\left(t_{k}\right) x\left(t_{k}\right)-\hat{x}\left(t_{k}^{-}\right)^{\prime} P\left(t_{k}^{-}\right) x\left(t_{k}^{-}\right)-c\left(t_{k}^{-}\right) \\
& \quad-\sum_{j \in \mathcal{I}_{k}} \bar{d}_{j}\left(I-\frac{\mathbf{y}_{j} \mathbf{y}_{j}^{\prime}}{\left\|\mathbf{y}_{j}\right\|^{2}}\right) \bar{d}_{j}=0 .
\end{aligned}
$$

Replacing (26) in (27) and these two equations in (28), we conclude that (11)-(12), and (17) hold.

Notice that for $P_{1}:=P\left(t_{1}\right)=P\left(t_{1}^{-}\right)+W\left(t_{1}\right)>0$ because $P\left(t_{1}^{-}\right)>0$ as it was proved above, and $W\left(t_{i}\right) \geq 0$, $i=1, \ldots, k$. Therefore, replacing the initial condition (19) by

$$
J\left(z ; t_{1}\right)=\left(z-\hat{x}_{1}\right)^{\prime} P_{1}\left(z-\hat{x}_{1}\right), \quad z \in \mathbb{R}^{n}
$$

with $\hat{x}_{1}=\hat{x}\left(t_{1}\right)$, and solving the linear partial differential equation (18), we conclude that (9)-(11) hold for $0 \leq t<$ $t_{2}$. Applying this line of reasoning successively until $i=k$ we conclude that (15) holds and from this that $\hat{x}(t)$ given by (8)-(12) is indeed the solution to Problem 1 . 


\section{ESTIMATOR CONVERGENCE}

In this section we investigate under what conditions the estimate $\hat{x}$ provided by Theorem 1 converges to the true state $x$ of the perspective system. The following technical assumptions are needed:

Assumption 1: There exist positive constants $\delta, \Delta \in$ $(0, \infty)$ such that $\delta I \leq G(u) G(u)^{\prime} \leq \Delta I, \forall_{u} \in \mathbb{R}^{n_{u}}$

Assumption 2: Let $\operatorname{Num}(t, \sigma), 0 \leq \sigma<t$ denote the number of time instants at which measurement arrive in the open interval $(\sigma, t)$. There exist finite positive constants $\tau_{D}$ and $N_{0}$, for which the following condition holds:

$$
\operatorname{Num}(t, \sigma) \leq N_{0}+\frac{t-\sigma}{\tau_{D}} .
$$

The constant $\tau_{D}$ is called the average dwell-time and $N_{0}$ the chatter bound.

Assumption 1 is a mild assumption and essentially guarantees that $G(u)$ is bounded and full-row rank, "uniformly" over all possible inputs. Assumption 2 roughly speaking guarantees that the average interval between consecutive arrival of measurements is no less than $\tau_{D}$. This type of condition typically arises in the context of logic-based switching control (cf., e.g., [19] and references therein). In our context it guarantees that the summation in (7) will not grow unbounded due to "too frequent" measurements. This assumption is purely technical and is need to simplify the analysis. In practice it always holds.

The following result establishes the convergence of the state estimate.

Theorem 2: Assuming that the solution to the process (1), (5) exists globally, the solution to the impulse state estimator (8)-(12) also exists globally. Moreover, when Assumptions 1-2 hold and $P^{-1}$ remains uniformly bounded, there exist positive constants $c, r<1, \gamma_{d}, \gamma_{1}, \ldots, \gamma_{N}$ such that

$$
\begin{aligned}
\left\|\tilde{x}\left(t_{k}\right)\right\| \leq c r^{k}\|\tilde{x}(0)\|+\gamma_{d} \sup _{\tau \in\left(0, t_{k}\right)}\|\mathbf{d}(\tau)\| \\
\quad+\sum_{j=1}^{N} \gamma_{j} \sup _{\tau \in\left(0, t_{k}\right)}\left\|\overline{\mathbf{n}}_{j}(\tau)\right\|, \quad t_{k}>0
\end{aligned}
$$

where $\tilde{x}(t):=\hat{x}(t)-x(t)$ denotes the state estimation error.

Proof: From (1) and (10) we conclude that for all $t_{i} \leq t<t_{i+1}$, the state estimation error evolves according to

$$
\dot{\tilde{x}}=A(u) \tilde{x}-G(u) \mathbf{d} .
$$

Defining,

$$
V:=\tilde{x} P \tilde{x},
$$

and computing its time derivative, it follows that

$$
\begin{aligned}
\dot{V} & =\tilde{x}^{\prime}\left(\dot{P}+P A+A^{\prime} P\right) \tilde{x}-2 \tilde{x} P G \mathbf{d} \\
& =-\tilde{x}^{\prime}\left(P G G^{\prime} P\right) \tilde{x}-2 \tilde{x}^{\prime} P G \mathbf{d}, \quad t_{i} \leq t<t_{i+1}
\end{aligned}
$$

By completing the squares and using Assumption 1, we conclude that

$$
\begin{aligned}
\dot{V} & =-\frac{1}{2} \tilde{x}^{\prime}\left(P G G^{\prime} P\right) \tilde{x}-\frac{1}{2}\left\|G^{\prime} P \tilde{x}+2 \mathbf{d}\right\|^{2}+2\|\mathbf{d}\|^{2} \\
& \leq-\frac{1}{2} \delta \lambda_{\min }(P) V+2\|\mathbf{d}\|^{2}, \quad t_{i} \leq t<t_{i+1}
\end{aligned}
$$

where $\lambda_{\min }(P)$ denotes the smallest eigenvalue of $P$. Using the assumption that $P$ is lower bounded by a positive value, and defining $\gamma:=\frac{1}{2} \delta \inf _{\tau \in\left[t_{i}, t_{i+1}\right)} \lambda_{\min }(P(\tau))$, we further conclude that for all $t_{i} \leq t<t_{i+1}$,

$$
V(t) \leq V\left(t_{i}\right) e^{-\gamma\left(t-t_{i}\right)}+\frac{2}{\gamma} \sup _{\tau \in\left[t_{i}, t\right)}\|\mathbf{d}(\tau)\|^{2} .
$$

Consider now $t=t_{i+1}$. From (11)-(12), the estimation error $\tilde{x}$ at time $t=t_{i+1}$ can be written as

$$
\begin{aligned}
\tilde{x}\left(t_{i+1}\right)=\left[I-P\left(t_{i+1}\right)^{-1} W\left(t_{i+1}\right)\right] & \tilde{x}\left(t_{i+1}^{-}\right) \\
& +P\left(t_{i+1}\right)^{-1} \eta,
\end{aligned}
$$

where $\eta:=\sum_{j \in \mathcal{I}_{i+1}} \bar{C}_{j}^{\prime} Y_{j}^{\prime} Y_{j} \overline{\mathbf{n}}_{j}$, and each $Y_{j}$ is a matrix for which $I-\frac{\mathbf{y}_{j} \mathbf{y}_{j}^{\prime}}{\left\|\mathbf{y}_{j}\right\|^{2}}=Y_{j}^{\prime} Y_{j}$. Such matrices always exist because $I-\frac{\mathbf{y}_{j} \mathbf{y}_{j}^{\prime}}{\left\|\mathbf{y}_{j}\right\|^{2}} \geq 0$. Thus, replacing (32) in (30), we get

$$
\begin{aligned}
& V\left(t_{i+1}\right)=\tilde{x}\left(t_{i+1}^{-}\right)^{\prime}\left[P\left(t_{i+1}\right)-2 W\left(t_{i+1}\right)\right. \\
& \left.+W\left(t_{i+1}\right) P\left(t_{i+1}\right)^{-1} W\left(t_{i+1}\right)\right] \tilde{x}\left(t_{i+1}^{-}\right) \\
& +\eta^{\prime} P\left(t_{i+1}\right)^{-1} \eta+2 \tilde{x}\left(t_{i+1}^{-}\right)^{\prime}\left[I-W\left(t_{i+1}\right) P\left(t_{i+1}\right)^{-1}\right] \eta .
\end{aligned}
$$

Observe that using (11) and resorting to the matrix inversion lemma ${ }^{1}$, and simplifying the notation by dropping the time dependence, $P\left(t_{i+1}\right)$ can be written as

$$
\begin{aligned}
P\left(t_{i+1}\right) & -2 W+W P\left(t_{i+1}\right)^{-1} W=P-W+W[P+W]^{-1} W \\
& =P-W^{\frac{1}{2}}\left[I-W^{\frac{1}{2}}\left[P+W^{\frac{1}{2}} I W^{\frac{1}{2}}\right]^{-1} W^{\frac{1}{2}}\right] W^{\frac{1}{2}} \\
& =P-W^{\frac{1}{2}} F W^{\frac{1}{2}}
\end{aligned}
$$

where $F:=\left[I+W^{\frac{1}{2}} P^{-1} W^{\frac{1}{2}}\right]^{-1}$ and $P=P\left(t_{i+1}^{-}\right)$. In this setting, given a positive semidefinite matrix $M$, we denote by $M^{\frac{1}{2}}$ any matrix such that $\left(M^{\frac{1}{2}}\right)^{\prime} M^{\frac{1}{2}}=M$. The others terms in (33) can be written as

$$
\begin{aligned}
P\left(t_{i+1}\right)^{-1}=(P+W)^{-1} & =P^{-1}-P^{-1} W^{\frac{1}{2}} F W^{\frac{1}{2}} P^{-1}, \\
I-W\left(t_{i+1}\right) P\left(t_{i+1}\right)^{-1} & =I-W^{\frac{1}{2}} I W^{\frac{1}{2}}\left[P+W^{\frac{1}{2}} I W^{\frac{1}{2}}\right]^{-1} \\
& =I-W^{\frac{1}{2}} F W^{\frac{1}{2}} P^{-1} .
\end{aligned}
$$

Thus,

$$
\begin{aligned}
V\left(t_{i+1}\right)= & \tilde{x}^{\prime} P \tilde{x}-\tilde{x}^{\prime} W^{\frac{1}{2}} F W^{\frac{1}{2}} \tilde{x} \\
& +\eta^{\prime}\left(P^{-1}-P^{-1} W^{\frac{1}{2}} F W^{\frac{1}{2}} P^{-1}\right) \eta \\
& +2 \tilde{x}^{\prime}\left(I-W^{\frac{1}{2}} F W^{\frac{1}{2}} P^{-1}\right) \eta .
\end{aligned}
$$

By completing the squares, we further conclude that

$$
V\left(t_{i+1}\right) \leq(1+\epsilon) V\left(t_{i+1}^{-}\right)+\left(1+\frac{1}{\epsilon}\right) \eta^{\prime} P^{-1} \eta,
$$

where $\epsilon$ is an arbitrary small positive constant. Therefore, resorting to (31), $V\left(t_{i+1}\right)$ satisfies

$$
V\left(t_{i+1}\right) \leq(1+\epsilon) V\left(t_{i}\right) e^{-\gamma\left(t_{i+1}-t_{i}\right)}+\frac{1}{\epsilon} a_{i+1}+b_{i+1},
$$

${ }^{1}$ Let $A, C$, and $A^{-1}+B^{\prime} C^{-1} B$ be non-singular matrices, then $\left(A^{-1}+\right.$ $\left.B^{\prime} C^{-1} B\right)^{-1}=A-A B^{\prime}\left(B A B^{\prime}+C\right)^{-1} B A$. Another useful matrix identity is the following $\left(A^{-1}+B^{\prime} C^{-1} B\right)^{-1} B^{\prime} C^{-1}=A B^{\prime}\left(B A B^{\prime}+\right.$ $C)^{-1}$. 
where

$$
\begin{aligned}
a_{i+1} & :=\lambda_{\max }\left(P^{-1}\right)\|\eta\|^{2} \\
b_{i+1} & :=(1+\epsilon) \frac{2}{\gamma} \sup _{\tau \in\left[t_{i}, t_{i+1}\right)}\|\mathbf{d}(\tau)\|^{2}+a_{i+1} .
\end{aligned}
$$

Furthermore, solving this inequality recursively, we get

$$
\begin{aligned}
V\left(t_{k}\right) \leq & (1+\epsilon)^{k} e^{-\gamma\left(t_{k}-t_{0}\right)} V\left(t_{0}\right) \\
& +\sum_{j=0}^{k-1}(1+\epsilon)^{j} e^{-\gamma\left(t_{k}-t_{k-j}\right)}\left(\frac{1}{\epsilon} a_{k-j}+b_{k-j}\right) .
\end{aligned}
$$

Applying Assumption 2, we first notice that $t_{k}-t_{k-j} \geq$ $\left[j-N_{0}\right] \tau_{D}, j=0,1, \ldots, k-1$. Consequently

$$
\begin{aligned}
V\left(t_{k}\right) & \leq\left[(1+\epsilon) e^{-\gamma \tau_{D}}\right]^{k} e^{\gamma N_{0} \tau_{D}} V\left(t_{0}\right) \\
+ & \sum_{j=0}^{k-1}\left[(1+\epsilon) e^{-\gamma \tau_{D}}\right]^{j}\left(\frac{1}{\epsilon} a_{k-j}+b_{k-j}\right) e^{\gamma N_{0} \tau_{D}}
\end{aligned}
$$

From this inequality, we further conclude that by picking $\epsilon>0$ such that $r:=(1+\epsilon) e^{-\gamma \max _{j} \tau_{D}}<1$, it follows that $V$ is bounded and $V\left(t_{k}\right) \rightarrow \frac{1}{1-r}\left(\frac{1}{\epsilon} \max _{j} a_{j}+\right.$ $\left.\max _{j} b_{j}\right) e^{\gamma \max _{j}\left\{N_{0} \tau_{D}\right\}}$ as $k \rightarrow \infty$. Since for every finite time $P$ is positive definite, $V$ must be finite on any finite interval and therefore so must be $\tilde{x}$ and $\hat{x}$. Global existence of solution follows. It is also straightforward to conclude from (34) that the ISS-like bound (29) holds.

Some condition on the observability of (1), (5) would be expected to achieve convergence of the estimated state $\hat{x}$ to the process state $x$. In Theorem 2 this condition appear in the form of the requirement that $P^{-1}$ remains bounded. The following result provides a condition under which this happens.

Lemma 1: The matrix $P^{-1}$ remains uniformly bounded along trajectories of the system (1), (5), and the stateestimator (8)-(12), provided that there exist positive constants $\mathbf{N}, \epsilon$ such that the following persistence of excitation condition

$$
\left.\frac{1}{\mathbf{N}} \sum_{j=0}^{\mathbf{N}} \Phi\left(t_{i+j}, t_{i}\right)^{\prime} W\left(t_{i+j}\right) \Phi\left(t_{i+j}, t_{i}\right)\right) \geq \epsilon I>0,
$$

$i=0,1, \ldots, k$, holds, where $\Phi(t, \tau)$ denotes the state transition matrix of $\dot{z}=A(u) z$.

Proof: Due to space limitations, the proof is omitted. It can be found in [20]

Combining Theorem 2 and Lemma 1 we obtain the following:

Corollary 1: When Assumptions 1 and 2 hold, and there exist constants $\mathbf{N}, \epsilon$ such that the persistence of excitation condition (35) holds, the state-estimate $\hat{x}$ converges to the state $x$ in the absence of disturbance input and measurement noise. When the disturbance and noise are bounded but nonzero, $\hat{x}$ may not converge to $x$ but remains bounded away from it.
The theoretical results presented in the previous sections were experimentally validated by applying them to estimate the position and orientation of a mobile robot using measurements from an on-board CCD camera. This section describes the experimental setup and presents the results obtained.

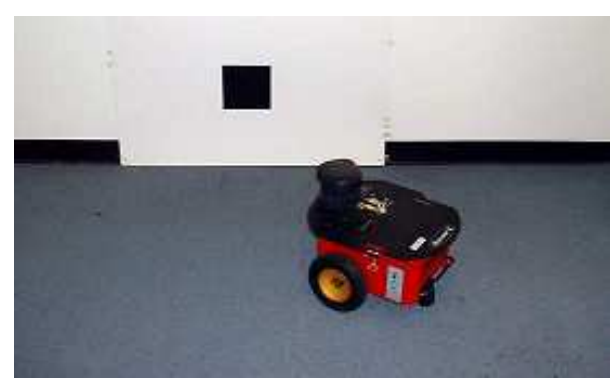

Fig. 1. Experimental setup: Pionner 2-DXE mobile robot with CCD camera mounted on top and visual landmark.

The experiments were carried out on a Pionner 2-DXE mobile robot from ActivMedia [21]. The vehicle, shown in Fig. 1, has two rear wheels which are powered by two independent high torque, reversible-DC motors, and one passive rear caster to balance the robot. The vehicle is equipped with a Sony EVI D30 pan-tilt-zoom (PTZ) color video camera mounted on the top of the robot with its optical axis oriented towards the forward direction (when pan and tilt angles are zero).

To simplify the image processing, in these experiments we used the corners of a black square as visual landmarks (see Fig. 1). The location of these points were obtained by detecting the edges of the square and then computing their intersections. A pan controller was also implemented to keep as much as possible the visual landmark in the center of the image. As it is shown in [20], [22], system (1)-(2) describes the kinematic model of the mobile robot whose outputs are the homogeneous image coordinates of $N$ (in this case $N=4$ ) fixed points provided by an onboard camera.

To validate the minimum-energy state estimator, several tests were carried out. Due to space limitations we present here results for a single test in which the vehicle follows a circular path with linear velocity $v=0.06 \mathrm{~m} / \mathrm{s}$ and angular velocity $\omega=0.09 \mathrm{rad} / \mathrm{s}$. Since the robot is describing a circular trajectory and the pan angle is limited to $\left[-\frac{\pi}{2}, \frac{\pi}{2}\right]$, the visual landmarks periodically left the camera's field of view. While this happened, the estimator did not receive any visual measurements. Fig. 2-4 show the experimental results. We can see that the output of the estimator converges to the values correspondent to a circular trajectory. Observe also the time evolution of the minimum and maximum singular values of $P$ when the estimator is receiving $(\gamma=0)$ or not receiving $(\gamma=1)$ measurements from the camera. Another interesting observation is the behavior of the pan controller that is always trying to compensate the motion of the robot in order to keep the features in the image. 

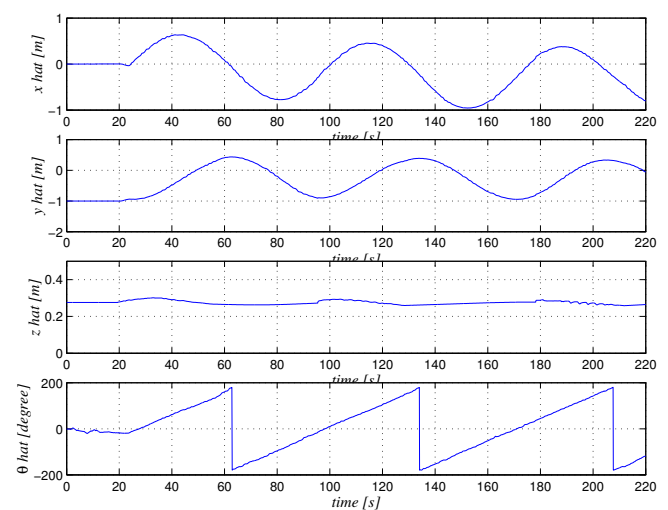

Fig. 2. Time evolution of the estimated position $(\hat{x}, \hat{y}, \hat{z})$, and orientation $\hat{\theta}$.

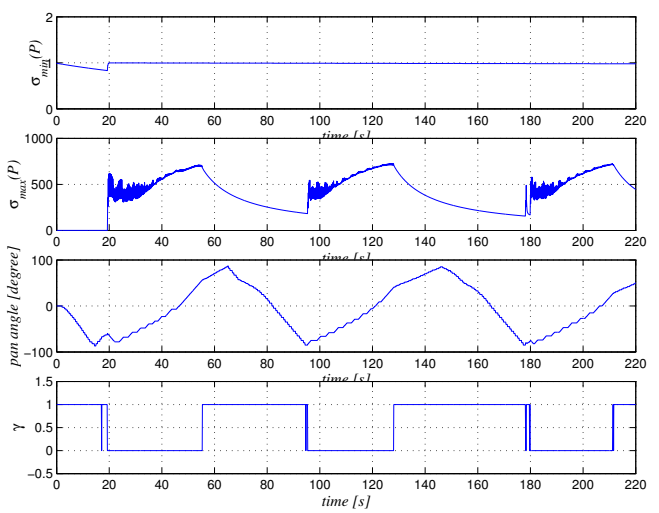

Fig. 3. Time evolution of the minimum and maximum singular values of $P$, respectively; the pan angle; and the variable $\gamma$ which indicates when the estimator is receiving $(\gamma=0)$ or not $(\gamma=1)$ measurements from the camera.

\section{CONCLUSIONS}

We considered the problem of estimating the state of continuous-time systems with perspective outputs, whose measurements arrive at discrete-time instants, are timedelayed, noisy, and may not be complete. We designed a dynamical impulsive system that produces an estimate of the state that is "most compatible" with the dynamics, in the sense that it requires the least amount of noise energy to explain the measured output. Under suitable observability assumptions, the state-estimate converges asymptotically to the true value of the state in the absence of noise and disturbance. In the presence of noise, the estimate remains bounded away from the true value of the state. We apply these results to the estimation of position and orientation for a mobile robot that use a monocular charged-coupleddevice (CCD) camera mounted on-board that observes the apparent motion of stationary points. Future work will address the application of these results to design output feedback controllers for autonomous vehicles.

\section{REFERENCES}

[1] B. K. Ghosh, M. Jankovic, and Y. T. Wu, "Perspective problems in system theory and its application in machine vision," J. Mathematical Syst. Estimation Contr., vol. 4, no. 1, pp. 3-38, 1994.

[2] B. K. Ghosh and E. P. Loucks, "A perspective theory for motion and shape estimation in machine vision," SIAM J. Contr. Optimization, vol. 33, no. 5, pp. 1530-1559, 1995.

[3] S. Takahashi and B. K. Ghosh, "Motion and shape parameters identification with vision and range," in Proc. of the 2001 Amer. Contr. Conf., vol. 6, June 2001, pp. 4626-4631.
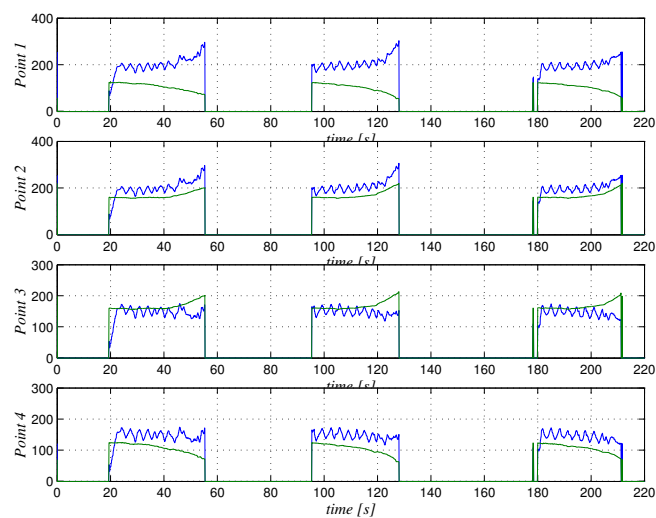

Fig. 4. Time evolution of the position in the image of the corners of the visual landmark. When the points are out of the camera's field of view, the points' coordinates are not shown.

[4] L. Matthies, T. Kanade, and R. Szeliski, "Kalman filter-based algorithms for estimating depth from image sequences," Int. J. of Comput. Vision, vol. 3, pp. 209-236, 1989.

[5] M. Jankovic and Ghosh, "Visually guided ranging from observations of points, lines and curves via an identifier based nonlinear observer," Syst. \& Contr. Lett., vol. 25, pp. 63-73, 1995.

[6] S. Soatto, R. Frezza, and P. Perona, "Motion estimation via dynamic vision," IEEE Trans. on Automat. Contr., vol. 41, no. 3, pp. 393-413, Mar. 1996.

[7] I. Kaminer, A. M. Pascoal, W. Kang, and O. Yakimenko, "Integrated vision/inertial navigation systems design using nonlinear filtering," IEEE Trans. Aerospace and Electronic Syst., vol. 37, no. 1, pp. 158172, Jan. 2001.

[8] A. Chiuso, P. Favaro, H. Jin, and S. Soatto, "Structure from motion causally integrated over time," IEEE Trans. on Pattern Anal. Mach. Intell., vol. 24, no. 4, pp. 523-535, Apr. 2002

[9] H. Rehbinder and B. K. Ghosh, "Pose estimation using line-based dynamic vision and inertial sensors," IEEE Trans. on Automat. Contr., vol. 48, no. 2, pp. 186-199, Feb. 2003.

[10] Y. Ma, J. Kosecka, and S. Sastry, "Vision guided navigation for a nonholonomic mobile robot," IEEE Trans. Robot. Automat., vol. 15, no. 3, pp. 521-536, 1999.

[11] O. Shakernia, Y. Ma, T. Koo, and S. Sastry, "Landing an unmanned air vehicle: vision based motion estimation and nonlinear control," Asian J. Contr., vol. 1, no. 3, pp. 128-145, 1999.

[12] J. Hespanha, O. Yakimenko, I. Kaminer, and A. Pascoal, "Linear parametrically varying systems with brief instabilities: an application to integrated vision/IMU navigation," in Proc. of the 40th Conf. on Decision and Contr., Dec. 2001.

[13] R. E. Mortensen, "Maximum likelihood recursive nonlinear filtering," J. Opt. Theory and Applications, vol. 2, pp. 386-394, 1968.

[14] O. J. Hijab, "Minimum energy estimation," Ph.D. dissertation, University of California, Berkeley, 1980.

[15] W. M. McEneaney, "Robust/H-infinity filtering for nonlinear systems," Syst. \& Contr. Lett., no. 33, pp. 315-325, 1998.

[16] A. J. Krener, "The convergence of the minimum energy estimator," in New Trends in Nonlinear Dynamics and Control, and Their Applications, ser. Lecture Notes in Control and Information Sciences, W. Kang, Ed. Springer-Verlag, 2003.

[17] J. P. Hespanha, "State estimation and control for systems with perspective outputs," in Proc. of the 41st Conf. on Decision and Contr., Dec. 2002.

[18] A. P. Aguiar and J. P. Hespanha, "Minimum-energy state estimation for systems with perspective outputs and state constraints," in Proc. of the 42nd Conf. on Decision and Contr., Dec. 2003.

[19] J. P. Hespanha and A. S. Morse, "Stability of switched systems with average dwell-time," in Proc. of the 38th Conf. on Decision and Contr., Dec. 1999.

[20] A. P. Aguiar and J. P. Hespanha, "State estimation for continuoustime systems with perspective outputs from discrete noisy timedelayed measurements," University of California, Santa Barbara, Tech. Rep., Mar. 2004.

[21] ActivMedia. [Online]. Available: http://www.activmedia.com/

[22] A. P. Aguiar and J. P. Hespanha, "Pose estimation of autonomous vehicles using visual information: A minimum-energy estimator approach," in Proc. of IAV2004 - 5th IFAC/EURON Symp. on Intel. Auton. Vehicles, July 2004. 